\title{
Polynomially compact derivations on Banach algebras
}

\author{
by \\ MateJ BrešAr (Maribor) and Yuri V. Turovski (Baku)
}

\begin{abstract}
We consider a continuous derivation $D$ on a Banach algebra $\mathcal{A}$ such that $p(D)$ is a compact operator for some polynomial $p$. It is shown that either $\mathcal{A}$ has a nonzero finite-dimensional ideal not contained in the $\operatorname{radical} \operatorname{rad}(\mathcal{A})$ of $\mathcal{A}$ or there exists another polynomial $\widetilde{p}$ such that $\widetilde{p}(D)$ maps $\mathcal{A}$ into $\operatorname{rad}(\mathcal{A})$. A special case where $D^{n}$ is compact is discussed in greater detail.
\end{abstract}

1. Introduction. In this paper we continue the line of investigation initiated in [4] and [5]. In [4] we considered compact derivations (and some slightly more general maps) on Banach algebras, and in [5] we considered compact elementary operators on Banach algebras. The reader is referred to these two papers for the history of these topics.

The purpose of the present paper is to treat the condition that a continuous derivation $D$ of a Banach algebra $\mathcal{A}$ is polynomially compact, i.e. $p(D)$ is a compact operator for some polynomial $p$. This is of course a considerably more general condition than the one considered in [4], and perhaps also a more natural one since nontrivial examples of polynomially compact derivations appear in some basic examples of Banach algebras (like $B(X)$ ) in which nonzero compact derivations do not exist. If $D$ is an inner derivation, then $p(D)$ is an elementary operator, so our condition is also connected with the one treated in [5]. Actually, it turns out that some results from [5] are applicable to problems treated here.

In Section 2 we show that for every polynomial $p$ there exists a polynomial $\widetilde{p}$ such that the condition that $p(D)$ is compact implies that $\widetilde{p}(D)$ maps $\mathcal{A}$ into its Jacobson $\operatorname{radical} \operatorname{rad}(\mathcal{A})$, unless $\mathcal{A}$ has finite-dimensional ideals not contained in $\operatorname{rad}(\mathcal{A})$ (and hence the compactness conditions may occur in a trivial fashion). In Section 3 we consider a special situation where $D^{n}$ is a compact operator. We obtain a rather definitive result for such derivations

2000 Mathematics Subject Classification: 47B47, 47B48, 47B07, 46H15, 46H20.

Key words and phrases: polynomially compact derivation, power compact derivation, Banach algebra, radical, minimal idempotent.

The first author is supported by ARRS Grant P1-0288. 
in the context of prime Banach algebras, which can be viewed as an analytic version of algebraic results $[2,3]$ dealing with the situation where $D^{n}$ has finite rank.

2. Polynomially compact derivations. By an "algebra" we shall always mean an associative algebra over $\mathbb{C}$. The first lemmas clearly hold in a somewhat more general setting, but we shall not bother the reader with technical statements.

To each polynomial $p \in \mathbb{C}[t]$ of degree $\geq 1$ and with leading coefficient 1 we attribute another polynomial $\widetilde{p} \in \mathbb{C}[t]$ as follows. If

$$
p(t)=\prod_{i=1}^{r}\left(t-\lambda_{i}\right)^{k_{i}},
$$

where $\lambda_{i} \in \mathbb{C}$ are pairwise distinct and $k_{i}$ are positive integers, then let

$$
\widetilde{p}(t)=\prod_{i=1}^{r} \prod_{j=1}^{r}\left(t-\lambda_{i}+\lambda_{j}\right)^{k_{i}+k_{j}-1} .
$$

Let $n=k_{1}+\cdots+k_{r}$ be the degree of $p$. Then the degree of $\widetilde{p}$ is $\widetilde{n}=2 r n-r^{2}$. Therefore $2 n-1 \leq \widetilde{n} \leq n^{2}$.

Until the end of this section let $p$ be a fixed polynomial. We are interested in the condition that $p(D)$ is a compact operator for some derivation $D$ on a Banach algebra $\mathcal{A}$ (of course, our restrictions that $n \geq 1$ and the leading coefficient of $p$ is 1 do not cause any loss of generality). Our approach is based on the following two elementary algebraic lemmas.

LEMma 2.1. Let $u_{1}, \ldots, u_{r}, v_{1}, \ldots, v_{r}$ be elements in a commutative algebra $\mathcal{C}$, and let $k_{1}, \ldots, k_{r}$ be positive integers. Then

$$
\prod_{i=1}^{r} \prod_{j=1}^{r}\left(u_{i}+v_{j}\right)^{k_{i}+k_{j}-1} \in u_{1}^{k_{1}} \cdots u_{r}^{k_{r}} \mathcal{C}+v_{1}^{k_{1}} \cdots v_{r}^{k_{r}} \mathcal{C} .
$$

Proof. Using the binomial formula we see that

$$
\left(u_{i}+v_{j}\right)^{k_{i}+k_{j}-1}=u_{i}^{k_{i}} a_{i j}+v_{j}^{k_{j}} b_{i j}
$$

for some $a_{i j}, b_{i j} \in \mathcal{C}$. This proves the lemma for $r=1$. Noting that

$$
\begin{aligned}
& \left(u_{r}+v_{r}\right)^{2 k_{r}-1} \cdot \prod_{i=1}^{r-1}\left(u_{i}+v_{r}\right)^{k_{i}+k_{r}-1} \cdot \prod_{j=1}^{r-1}\left(u_{r}+v_{j}\right)^{k_{r}+k_{j}-1} \\
& \quad=\left(u_{r}^{k_{r}} a_{r r}+v_{r}^{k_{r}} b_{r r}\right) \cdot \prod_{i=1}^{r-1}\left(u_{i}^{k_{i}} a_{i r}+v_{r}^{k_{r}} b_{i r}\right) \cdot \prod_{j=1}^{r-1}\left(u_{r}^{k_{r}} a_{r j}+v_{j}^{k_{j}} b_{r j}\right)
\end{aligned}
$$




$$
\begin{aligned}
& \in u_{r}^{k_{r}} \cdot \prod_{i=1}^{r-1}\left(u_{i}^{k_{i}} a_{i r}+v_{r}^{k_{r}} b_{i r}\right) \cdot \mathcal{C}+v_{r}^{k_{r}} \cdot \prod_{j=1}^{r-1}\left(u_{r}^{k_{r}} a_{r j}+v_{j}^{k_{j}} b_{r j}\right) \cdot \mathcal{C} \\
& \subseteq u_{r}^{k_{r}} v_{r}^{k_{r}} \mathcal{C}+u_{1}^{k_{1}} \cdots u_{r}^{k_{r}} \mathcal{C}+v_{1}^{k_{1}} \cdots v_{r}^{k_{r}} \mathcal{C}
\end{aligned}
$$

one easily completes the proof by induction on $r$.

Let $\mathcal{A}$ be an algebra. For $a, b \in \mathcal{A}$ we define operators $L_{a}, R_{b}: \mathcal{A} \rightarrow \mathcal{A}$ by $L_{a}(x)=a x$ and $R_{b}(x)=x b$. If $\mathcal{A}$ does not have an identity element, then these definitions clearly make sense also when $a$ and $b$ belong to the algebra obtained from $\mathcal{A}$ by adjoining 1 to $\mathcal{A}$. In particular, $L_{1}$ is the identity operator $I$. Obviously, $L_{a}$ and $R_{b}$ always commute. Further, we set ad $a=$ $L_{a}-R_{a}$, so that ad $a(x)=[a, x]$.

Lemma 2.2. Let $\mathcal{B}$ be an algebra, let $b \in \mathcal{B}$ and let $\mathcal{C}$ be the algebra of operators on $\mathcal{B}$ generated by $L_{b}, R_{b}$ and $I$. Then there exist $E, F \in \mathcal{C}$ such that $\widetilde{p}(\operatorname{ad} b)=L_{p(b)} E+R_{p(b)} F$.

Proof. We have

$$
\widetilde{p}(\operatorname{ad} b)=\widetilde{p}\left(L_{b}-R_{b}\right)=\prod_{i=1}^{r} \prod_{j=1}^{r}\left(L_{b-\lambda_{i}}-R_{b-\lambda_{j}}\right)^{k_{i}+k_{j}-1} .
$$

We are now in a position to apply Lemma 2.1 with $L_{b-\lambda_{i}}$ playing the role of $u_{i}$ and $-R_{b-\lambda_{j}}$ playing the role of $v_{j}$. Hence there exist $E, F \in \mathcal{C}$ such that

$$
\begin{aligned}
& \widetilde{p}(\operatorname{ad} b)=L_{b-\lambda_{1}}^{k_{1}} \cdots L_{b-\lambda_{r}}^{k_{r}} E+R_{b-\lambda_{1}}^{k_{1}} \cdots R_{b-\lambda_{r}}^{k_{r}} F \\
& =L_{\left(b-\lambda_{1}\right)^{k_{1}}} \cdots L_{\left(b-\lambda_{r}\right)^{k_{r}}} E+R_{\left(b-\lambda_{1}\right)^{k_{1}}} \cdots R_{\left(b-\lambda_{r}\right)^{k_{r}}} F \\
& =L_{p(b)} E+R_{p(b)} F \text {. }
\end{aligned}
$$

It should be mentioned that the idea from [8] is hidden in the proof of Lemma 2.2; however, this idea is modified and refined.

LEMMA 2.3. Let $D$ be a continuous derivation on a Banach algebra $\mathcal{A}$. Suppose that $p(D)$ is a compact operator. Then $\widetilde{p}(\operatorname{ad} D)(T)$ is a compact operator for every continuous linear operator $T: \mathcal{A} \rightarrow \mathcal{A}$.

Proof. We apply Lemma 2.2 for the case where $\mathcal{B}$ is the algebra of all continuous linear operators on $\mathcal{A}$, and $b=D$. Therefore there exist $A_{i}, B_{i}, C_{j}, D_{j}$ in the subalgebra generated by $D$ and $I$ such that

$$
\widetilde{p}(\operatorname{ad} D)(T)=\sum_{i} p(D) A_{i} T B_{i}+\sum_{j} C_{j} T D_{j} p(D) .
$$

Since $p(D)$ is compact, so is $\widetilde{p}(\operatorname{ad} D)(T)$.

The meaning of Lemma 2.3 will become clear in the next theorem. In its proof we shall see that the lemma gives rise to completely continuous 
elements in $\mathcal{A}$, i.e. elements such $a$ that both $L_{a}$ and $R_{a}$ are compact operators.

TheOREM 2.4. Let $D$ be a continuous derivation on a Banach algebra $\mathcal{A}$. If $p(D)$ is a compact operator, then either $\widetilde{p}(D)$ maps $\mathcal{A}$ into $\operatorname{rad}(\mathcal{A})$, or $\mathcal{A}$ has a nonzero finite-dimensional ideal which is not contained in $\operatorname{rad}(\mathcal{A})$.

Proof. The condition that $D$ is a derivation can be expressed as

$$
\text { ad } D\left(L_{x}\right)=L_{D(x)}, \quad \text { ad } D\left(R_{x}\right)=R_{D(x)}
$$

for every $x \in \mathcal{A}$. This readily implies that

$$
\widetilde{p}(\operatorname{ad} D)\left(L_{x}\right)=L_{\widetilde{p}(D)(x)}, \quad \widetilde{p}(\operatorname{ad} D)\left(R_{x}\right)=R_{\widetilde{p}(D)(x)} .
$$

Lemma 2.3 now tells us that $L_{\widetilde{p}(D)(x)}$ and $R_{\widetilde{p}(D)(x)}$ are compact operators; that is to say, $\widetilde{p}(D)(x)$ is a completely continuous element for every $x \in \mathcal{A}$. Assuming that $\widetilde{p}(D)$ does not map $\mathcal{A}$ into $\operatorname{rad}(\mathcal{A})$ it follows that $\mathcal{A}$ contains completely continuous elements that are not contained in $\operatorname{rad}(\mathcal{A})$. But then $\mathcal{A}$ contains a nonzero finite-dimensional ideal not contained in $\operatorname{rad}(\mathcal{A})$ [5, Corollary 3.3].

If we add to Theorem 2.4 the assumption that $\mathcal{A}$ does not contain nonzero finite-dimensional nilpotent ideals (for example, if $\mathcal{A}$ is semiprime), then more can be said - namely, $\mathcal{A}$ then contains a nonzero central idempotent $e$ such that $e \mathcal{A}$ is a finite-dimensional ideal [5, Corollary 3.4]. In particular, if $\mathcal{A}$ is prime, i.e. it does not contain nonzero ideals whose product is zero, then $\mathcal{A}$ itself must be finite-dimensional, since 0 and 1 are the only possible central idempotents in $\mathcal{A}$. Thus, the following corollary holds.

Corollary 2.5. Let $D$ be a continuous derivation on an infinite-dimensional prime Banach algebra $\mathcal{A}$. If $p(D)$ is a compact operator, then $\widetilde{p}(D)$ maps $A$ into $\operatorname{rad}(\mathcal{A})$.

We remark that there exist nonzero compact derivations on infinitedimensional prime algebras that have their range in the radical $[4$, p. 136]. Thus the situation described in Corollary 2.5 can appear in a nontrivial fashion even for $p(t)=t$.

Assuming additionally that $\mathcal{A}$ in Corollary 2.5 is semisimple, the conclusion reads as $\widetilde{p}(D)=0$. In particular, $D$ is then an algebraic derivation. A simple example of a semisimple, prime and infinite-dimensional Banach algebra is $B(X)$, the algebra of all bounded linear operators on an infinitedimensional Banach space $X$. Thus, only algebraic derivations on $B(X)$ can be polynomially compact.

3. Power compact derivations. We continue the discussion from the previous section, restricting our attention to the polynomial $p(t)=t^{n}$. Note that then $\widetilde{p}(t)=t^{2 n-1}$. 
By Corollary 2.5 we know that if $D$ is a continuous derivation on an infinite-dimensional prime Banach algebra $\mathcal{A}$ such that $D^{n}$ is compact, then $D^{2 n-1}$ maps $\mathcal{A}$ into $\operatorname{rad}(\mathcal{A})$. In particular, $D^{2 n-1}$ must be 0 if $\mathcal{A}$ is semisimple. Let us point out an example where such a situation occurs.

EXAmple 3.1. Let $\mathcal{A}=\mathcal{B}(X)$ where $X$ is an infinite-dimensional Banach space. Let $T \in \mathcal{B}(X)$ be such that $T^{m}=0$ and $T^{k}$ is a compact operator for some $1 \leq k<m$. Consider the inner derivation $D=\operatorname{ad} T$. Note that

$$
D^{m+k-1}=\left(L_{T}-R_{T}\right)^{m+k-1}=\sum_{i=0}^{m+k-1}(-1)^{i}\left(\begin{array}{c}
m+k-1 \\
i
\end{array}\right) L_{T^{m+k-1-i}} R_{T^{i}} .
$$

As $T^{m}=0$, this sum reduces to

$$
D^{m+k-1}=\sum_{i=k}^{m-1}(-1)^{i}\left(\begin{array}{c}
m+k-1 \\
i
\end{array}\right) L_{T^{m+k-1-i}} R_{T^{i}} .
$$

Since $T^{i}$ and $T^{m+k-1-i}$ are compact operators on $X$ for $i=k, \ldots, m-1$, it follows that $L_{T^{m+k-1-i}} R_{T^{i}}$ is a compact operator on $\mathcal{A}$ (see e.g. [1, Theorem 3(i), p. 174]). But then $D^{m+k-1}$ is also a compact operator on $\mathcal{A}$.

We moreover remark that $T^{m}=0$ implies $D^{2 m-1}=0$.

The goal of this section is to show that this example of a power compact derivation is essentially the only nontrivial example in the context of prime algebras (Theorem 3.3). In the proof we need the following lemma. Let us first recall that an element $a$ in a Banach algebra $\mathcal{A}$ is said to be compact if $L_{a} R_{a}$ is a compact operator.

Lemma $3.2([3])$. Let $D$ be a derivation of a Banach algebra $\mathcal{A}$. If $D^{n}$ is a compact operator, then the range of $D^{n}$ consists of compact elements.

The statement of Lemma 3.2 is explicitly mentioned at the very end of [3]. This is actually a simple consequence of a purely algebraic result on higher commutators in rings [3, Theorem 1.1]. Its proof, however, is rather long and involved.

TheOREM 3.3. Let $\mathcal{A}$ be an infinite-dimensional prime Banach algebra, let $D$ be a derivation of $\mathcal{A}$ and let $n$ be a positive integer. If $D^{n}$ is a compact operator whose range is not contained in $\operatorname{rad}(\mathcal{A})$, then $n \geq 2$ and $\mathcal{A}$ is a primitive algebra containing a minimal idempotent $e$. Moreover, if $\pi$ is the regular representation of $\mathcal{A}$ on the Banach space $X=\mathcal{A} e$, then there exist $T \in \mathcal{B}(X)$ and an integer $s$ such that $\pi(D(a))=T \pi(a)-\pi(a) T$ for all $a \in \mathcal{A},(n-1) / 2<s<n, T^{s+1}=0, T^{s} \neq 0$, and $T^{n-s}$ is a compact operator.

Proof. Lemma 3.2 tells us in particular that $\mathcal{A}$ contains compact elements which are not contained in $\operatorname{rad}(\mathcal{A})$. But then $\mathcal{A}$ contains a minimal 
idempotent $e$ by [5, Corollary 5.2]. Clearly, $X=\mathcal{A} e$ is a Banach space and the regular representation $\pi$ of $\mathcal{A}$ on $X$ (defined by $\pi(a) x e=a x e$ for all $x e \in X$ ) is faithful (since $\mathcal{A}$ is prime) and irreducible (since $\mathcal{A} e$ is a minimal left ideal of $\mathcal{A}$ ). Therefore $\mathcal{A}$ is a primitive, and hence also a semisimple algebra. As is well-known, $\pi(\mathcal{A})$ is a dense subalgebra of $\mathcal{B}(X)$ (see e.g. [1, Corollary 5, p. 128]).

Define $T_{0}: X \rightarrow X$ by

$$
T_{0} x e=D(x e) e .
$$

Since $D$, being a derivation on a semisimple Banach algebra, is automatically continuous [6], it follows that $T_{0} \in \mathcal{B}(X)$. For every $a \in \mathcal{A}$ we have

$$
\begin{aligned}
\left(T_{0} \pi(a)-\pi(a) T_{0}\right) x e & =T_{0} a x e-a\left(T_{0} x e\right) \\
& =D(a x e) e-a D(x e) e=D(a) x e=\pi(D(a)) x e
\end{aligned}
$$

for every $x e \in X$, showing that $\pi(D(a))=T_{0} \pi(a)-\pi(a) T_{0}$.

As mentioned at the beginning of this section, Corollary 2.5 implies that $D^{2 n-1}=0$. In particular, this shows that $n \neq 1$. Further, the derivation $D_{\pi}=\pi D \pi^{-1}=\operatorname{ad} T_{0}$ of the algebra $\pi(\mathcal{A})$ then also satisfies $D_{\pi}^{2 n-1}=0$. We claim that this implies that $\left(T_{0}-\lambda\right)^{n}=0$ for some $\lambda \in \mathbb{C}$. If $T_{0}$ lies in $\pi(\mathcal{A})$, then this follows immediately from [7, Corollary 2]; fortunately, the same proof works even when $T_{0} \in B(X) \backslash \pi(\mathcal{A})$. Set $T=T_{0}-\lambda$. Clearly, $T \in \mathcal{B}(X), T^{n}=0$ and $\pi(D(a))=T \pi(a)-\pi(a) T$ for all $a \in \mathcal{A}$.

Let $s<n$ be such that $T^{s+1}=0$ and $T^{s} \neq 0$. It is easy to see that then $D^{2 s+1}=0$. Since $D^{n} \neq 0$, we must have $(n-1) / 2<s$. Pick $u \in \mathcal{A}$ such that $T^{s} u e \neq 0$. Since $T^{s+1}=0$ it follows that $T^{s} u e, T^{s-1} u e, \ldots, T^{n-s} u e$ are linearly independent vectors in $X$. By density there is $v \in \mathcal{A}$ such that $\pi(v) T^{s} u e=e$ and $\pi(v) T^{s-1} u e=\cdots=\pi(v) T^{n-s} u e=0$. Accordingly, for every $x \in \mathcal{A}$ we have

$$
\begin{aligned}
\left(R_{u e} D^{n} R_{v}\right) x e & =D^{n}(x e v) u e=\pi\left(D^{n}(x e v)\right) u e \\
& =\sum_{i=n-s}^{s}(-1)^{i}\left(\begin{array}{c}
n \\
i
\end{array}\right) T^{n-i} \pi(x e v) T^{i} u e \\
& =\sum_{i=n-s}^{s}(-1)^{i}\left(\begin{array}{c}
n \\
i
\end{array}\right) T^{n-i} \pi(x e) \pi(v) T^{i} u e \\
& =(-1)^{s}\left(\begin{array}{c}
n \\
s
\end{array}\right) T^{n-s} \pi(x e) e=(-1)^{s}\left(\begin{array}{c}
n \\
s
\end{array}\right) T^{n-s} x e .
\end{aligned}
$$

That is, $(-1)^{s}\left(\begin{array}{l}n \\ s\end{array}\right) T^{n-s}$ is equal to the restriction of $R_{u e} D^{n} R_{v}$ to $X$. Since $D^{n}$ is compact it follows that $T^{n-s}$ is compact.

If $\pi^{-1}$ is continuous then the converse to Theorem 3.3 holds true as well. Indeed, assuming that the conclusion of this theorem holds we see that the 
operators $T^{i}, i=n-s, \ldots, s$, are compact, hence $L_{T^{n-i}} R_{T^{i}}$ are compact operators on $\mathcal{B}(X)$, and so

$$
D^{n}=\pi^{-1}\left(\sum_{i=n-s}^{s}(-1)^{i}\left(\begin{array}{l}
n \\
i
\end{array}\right) L_{T^{n-i}} R_{T^{i}}\right) \pi
$$

is a compact operator; moreover, it is easy to see that $D^{n} \neq 0=\operatorname{rad}(\mathcal{A})$.

\section{References}

[1] F. F. Bonsall and J. Duncan, Complete Normed Algebras, Springer, New York, 1973.

[2] M. Brešar and D. Eremita, The lower socle and finite rank elementary operators, Comm. Algebra 31 (2003), 1485-1497.

[3] M. Brešar, D. Eremita and T.-L. Wong, On commutators and derivations in rings, J. Algebra 278 (2004), 704-724.

[4] M. Brešar and Yu. V. Turovskii, A note on compact semiderivations, in: Banach Center Publ. 67, Inst. Math., Polish Acad. Sci., Warszawa, 2005, 133-137.

[5] —, - Compactness conditions for elementary operators, Studia Math. 178 (2007), $1-18$.

[6] B. E. Johnson and A. M. Sinclair, Continuity of derivations and a problem of Kaplansky, Amer. J. Math. 90 (1968), 1067-1073.

[7] W. S. Martindale and C. R. Miers, On the iterates of derivations of prime rings, Pacific J. Math. 104 (1983), 179-190.

[8] Yu. V. Turovskii and V. S. Shul'man, Conditions for the massiveness of the range of a derivation of a Banach algebra and of associated differential operators, Mat. Zametki 42 (1987), 305-314 (in Russian); English transl.: Math. Notes 12 (1987), 669-674.

Department of Mathematics, FNM

University of Maribor

Koroška 160

2000 Maribor, Slovenia

E-mail: bresar@uni-mb.si
Institute of Mathematics and Mechanics National Academy of Sciences of Azerbaijan

F. Agaev St. 9

Baku AZ1141, Azerbaijan

E-mail: yuri.turovskii@gmail.com

Received January 21, 2008

Revised version July 26, 2008 